\title{
Novo método de classificação de coeficientes de variação para a cultura do arroz de terras altas ${ }^{(1)}$
}

\author{
Nilce Helena de Araújo Diniz Costa ${ }^{(2)}$, José Carlos Seraphin ${ }^{(2)}$ e Francisco José Pfeilsticker Zimmermann ${ }^{(3)}$
}

Resumo - A avaliação de coeficientes de variação (CV) como medida de precisão em experimentos tem sido realizada em diversas culturas, com alguns trabalhos propondo métodos para obtenção de faixas de classificação de CV, e outros trabalhos definindo as faixas de classificação, sempre com base na média e no desvio-padrão dos CVs, e pressupondo que os mesmos têm distribuição normal, o que nem sempre é verdadeiro. Este trabalho teve por objetivo propor um método para definir faixas de classificação de $\mathrm{CV}$, independentemente da distribuição que possam apresentar; o método é baseado no uso da mediana (Md) e do pseudo-sigma (PS). Utilizaram-se, para este estudo, dados de 110 experimentos com arroz de terras altas, delineados em blocos completos casualizados, blocos incompletos generalizados, e reticulados quadrados, selecionando-se os caracteres relacionados às doenças, ao acamamento, ao rendimento de grãos e a alguns componentes da produção. Foram construídas faixas de classificação de CV de cada caráter estudado, de forma geral e considerando o delineamento experimental empregado em experimentos com 30 a 100 tratamentos. O método proposto é eficiente para a definição de faixas dos CVs, independentemente de sua distribuição, e a variação nos valores do CV evidencia a importância de se considerar não só a variável em estudo, mas também o delineamento experimental utilizado. Experimentos que apresentaram menores $\mathrm{CV}$ s foram aqueles em que se empregaram os Delineamentos de Blocos Incompletos. As variáveis relacionadas às doenças e ao acamamento apresentam maiores coeficientes de variação.

Termos para indexação: Oryza sativa, experimentação, métodos de análise.

\section{A new method of variation coefficient classification for upland rice crop}

\begin{abstract}
The assessment of coefficients of variation (CV) as a measure of precision in experiments has been done for various crops, and some researchers have proposed a methodology to get classification bands of $\mathrm{CV}$, and others defining the classification bands itself; based on the mean and on the standard deviation of the $\mathrm{CV}$, assuming that they have normal distribution, which is not always true. This study had as objective to propose a methodology for CV classification bands definition, independently of their distribution; the new methodology is based on the use of the median and the pseudosigma. Data from 110 experiments of upland rice, designed in randomized complete blocks, generalized incomplete blocks and square lattices were analyzed, selecting the characters related to diseases, lodging, yield and yield components. CV classification bands were constructed for each character studied, in general, and considering the experimental design utilized for experiments with 30 to 100 treatments. The method proposed is efficient for the definition of CV bands, independently of their distribution, and the variation of the values of $\mathrm{CV}$ evidences the importance of considering, not only the variable in study, but also the experimental design. Experiments with smaller CV were those that utilized Incomplete Block Design. The variables related to diseases and lodging were those that presented larger coefficient of variation.
\end{abstract}

Index terms: Oryza sativa, experimentation, analytical methods.

\footnotetext{
(1) Aceito para publicação em 9 de maio de 2001.

(2) Universidade Federal de Goiás, Instituto de Matemática e Estatística, Caixa Postal 131, CEP 74001-970 Goiânia, GO. E-mail: nilcehelena@cultura.com.br, seraphin@mat.ufg.br

${ }^{(3)}$ Embrapa-Centro Nacional de Pesquisa de Arroz e Feijão, Caixa Postal 179, CEP 75375-000 Santo Antônio de Goiás, GO. E-mail: fjpz@cnpaf.embrapa.br
}

\section{Introdução}

O erro experimental que ocorre nos experimentos agrícolas é influenciado pela precisão com que os experimentos são conduzidos (Chaves, 1985). A priori, quanto menor o erro experimental, maior a 
precisão do experimento. Para a minimização do erro experimental, alguns fatores devem ser considerados nos experimentos agrícolas, como: o tamanho das parcelas, o número de repetições, o delineamento experimental utilizado, a heterogeneidade dos solos, e a cultura utilizada. À medida que se aumenta a precisão do experimento, menor diferença significativa será detectada entre as estimativas das médias (Estefanel et al., 1987).

A existência de um coeficiente que estime a precisão experimental é fundamental, uma vez que trabalhos científicos são realizados e comparados (Scapim et al., 1995). O coeficiente de variação (CV), definido como a estimativa do erro experimental em porcentagem da estimativa da média, é uma das medidas estatísticas mais utilizadas pelos pesquisadores na avaliação da precisão dos experimentos. Entretanto, saber se um coeficiente de variação em particular é excessivamente alto ou baixo requer experiência com dados similares (Steel \& Torrie, 1980).

A avaliação de coeficientes de variação, como medida de precisão em experimentos, tem sido realizada com relação a diversas culturas, com alguns trabalhos propondo métodos para obtenção de faixas de classificação de coeficientes de variação (Estefanel et al., 1987; Garcia, 1989; Amaral et al., 1997), e outros, definindo as faixas de classificação (Campos, 1984; Pimentel-Gomes, 1985; Scapim et al., 1995; Judice et al., 1999), com base na relação entre a média dos coeficientes de variação e o seu desvio-padrão.

Não há, entretanto, referências sobre valores de coeficientes de variação para a cultura do arroz de terras altas ou de sequeiro, apesar de esta constituir uma das mais importantes opções para a agricultura brasileira, considerando-se sua importância na dieta da população. Além disso, os métodos propostos para a construção de faixas pressupõem que os coeficientes de variação tenham distribuição normal, o que nem sempre é verdadeiro.

Este trabalho teve por objetivo avaliar a distribuição do coeficiente de variação em experimentos com arroz de terras altas, e propor um método para definir faixas de classificação de coeficientes de variação, independentemente da distribuição que estes possam apresentar, considerando-se que os dados nem sempre têm distribuição normal.

\section{Material e Métodos}

Foram utilizados dados de 110 experimentos de arroz de terras altas, provenientes de projetos de pesquisa sobre melhoramento genético do arroz de terras altas, liderados pela Embrapa-Centro Nacional de Pesquisa de Arroz e Feijão (Embrapa-CNPAF), localizada no Município de Santo Antônio de Goiás, GO, no período de 1991 a 1998. Estes experimentos foram realizados na maior parte das áreas produtoras de arroz de terras altas do País, localizadas nas regiões Sudeste, Centro-Oeste e Norte, agrupadas em regiões II e III, divisões regionais criadas pela Embrapa-CNPAF, para facilidade de organização (Embrapa, 1997).

Os ensaios conduzidos foram basicamente os comparativos preliminares (ECP) e avançados (ECA), delineados em blocos completos casualizados (BC), blocos incompletos e reticulados quadrados (BI), com diferentes números de tratamentos (genótipos de arroz), com três ou quatro repetições. Os experimentos com até 29 tratamentos utilizaram BC, e os experimentos com 30 a 100 tratamentos utilizaram BC ou BI (Tabela 1). Esses 110 ensaios também foram objeto de estudo em Costa (2000), o qual considerou o efeito do tipo de ensaio conduzido, da região de cultivo e do número de tratamentos empregado, e em Cunha (2000), que considerou o efeito das transformações de dados sobre o coeficiente de variação.

Os caracteres selecionados para estudo foram os que tiveram maior freqüência nos experimentos e que estavam relacionados com o objetivo original dos projetos, que era o de obter cultivares com alta estabilidade de produção, boa qualidade de grão e resistentes à seca, ao acamamento, às doenças e às pragas. Estes caracteres foram: acamamento (ACA), altura da planta (ALT), brusone na folha (BF), brusone na panícula (BP), escaldadura da folha (ESC), floração (FLO), mancha dos grãos (MG), mancha parda na folha (MP), massa de 100 grãos (M100), número de espiguetas vazias (NEV), número de grãos cheios (NGC), número de panículas (NPAN) e produção de grãos (PROD).

As estimativas dos coeficientes de variação experimental dos caracteres em estudo foram obtidas pelo processamento das análises de variância, de acordo com os modelos dos delineamentos empregados para os experimentos, utilizando a fórmula:

$\mathrm{CV}=100\left(\mathrm{QME}^{0,5 / \overline{\mathrm{X}}}\right)$,

onde, QME é o quadrado médio do resíduo; e $\overline{\mathrm{X}}$ é a estimativa da média.

O método para definição das faixas de classificação de coeficientes de variação baseou-se no uso da mediana (Md) 
e do pseudo-sigma (PS) (Tukey, 1977; Hoaglin et al., 1983; Blanxart et al., 1992), medidas estas mais resistentes que a média e o desvio-padrão. Os intervalos de coeficientes de variação foram então definidos da seguinte forma:

$$
\begin{aligned}
& \text { Baixo }=\mathrm{CV} \leq(\mathrm{Md}-1 \mathrm{PS}) \\
& \text { Médio }=(\mathrm{Md}-1 \mathrm{PS})<\mathrm{CV} \leq(\mathrm{Md}+1 \mathrm{PS}) \\
& \text { Alto }=(\mathrm{Md}+1 \mathrm{PS})<\mathrm{CV} \leq(\mathrm{Md}+2 \mathrm{PS}) \\
& \text { Muito alto }=\mathrm{CV}>(\mathrm{Md}+2 \mathrm{PS})
\end{aligned}
$$

onde: $\mathrm{Md}=\left(\mathrm{Q}_{1}+\mathrm{Q}_{3}\right) / 2$ é a mediana dos coeficientes de variação para $Q_{1}, Q_{3}$ primeiro e terceiro quartil respectivamente, os quais delimitam $25 \%$ de cada extremidade da distribuição e, $\mathrm{PS}=\mathrm{IQR} / 1,35$

é o pseudo-sigma para IQR, amplitude interquartílica, medida resistente que indica o quanto os dados estão distanciados da mediana.

O pseudo-sigma seria o desvio-padrão que uma distribuição normal precisaria ter a fim de produzir a mesma amplitude interquartílica como os dados utilizados. Esta interpretação do pseudo-sigma é justificada pela presença do valor 1,35 em (2) que é obtido a partir da distribuição normal e corresponde à distância entre $\mathrm{Q}^{1} \mathrm{e} \mathrm{Q}^{3}$, que equivale a $50 \%$ dos dados, deixando $25 \%$ em cada extremidade. Quando se divide IQR por 1,35 o resultado obtido produz o desvio-padrão que se esperaria que tivesse uma distribuição normal.
Quando os dados não têm distribuição normal, o uso do pseudo-sigma como uma medida de dispersão será mais resistente que o desvio-padrão (s) clássico; entretanto, se os dados têm distribuição aproximadamente normal, o pseudo-sigma produz uma estimativa de $\sigma$, que será bem próxima de s, que é o desvio-padrão da amostra (Hoaglin et al., 1983; Blanxart et al., 1992).

Sob a condição de normalidade, tem-se que $68,27 \%$ dos CVs estão incluídos entre Md $\pm 1 \mathrm{PS} ; 95,45 \%$ dos CVs estão incluídos entre $\mathrm{Md} \pm 2 \mathrm{PS}$; e 99,73\% dos CVs estão incluídos entre Md \pm 3PS (Spiegel, 1993). Portanto, as freqüências esperadas dos coeficientes de variação nos intervalos definidos em (1) correspondem a: 15,86\%, $68,27 \%, 13,59 \%$ e $2,28 \%$, respectivamente.

Com a finalidade de medir a eficiência do método proposto (1), foi feita uma analogia entre esta e o método de Garcia (1989), e, para tanto, foram utilizados os caracteres que apresentaram distribuição normal. Para verificar quais caracteres se ajustavam à curva normal, foi aplicado o teste de Lilliefors (Conover, 1971; Campos, 1979). O método de Garcia (1989) baseia-se na média dos coeficientes de variação (m) e no desvio-padrão (s), supondo normalidade dos dados, para a construção de intervalos de coeficientes de variação da seguinte forma:

$$
\begin{aligned}
& \text { Baixo }=\mathrm{CV} \leq(\mathrm{m}-1 \mathrm{~s}) \\
& \text { Médio }=(\mathrm{m}-1 \mathrm{~s})<\mathrm{CV} \leq(\mathrm{m}+1 \mathrm{~s}) \\
& \text { Alto }=(\mathrm{m}+1 \mathrm{~s})<\mathrm{CV} \leq(\mathrm{m}+2 \mathrm{~s}) \\
& \text { Muito alto }=\mathrm{CV}>(\mathrm{m}+2 \mathrm{~s})
\end{aligned}
$$

\begin{tabular}{|c|c|c|c|c|c|c|c|}
\hline \multirow[t]{4}{*}{ Variável } & \multirow{4}{*}{$\begin{array}{l}\text { Teste de } \\
\text { Lilliefors }\end{array}$} & \multicolumn{3}{|c|}{ Número de experimentos } & \multicolumn{3}{|c|}{ Coeficiente de variação (\%) } \\
\hline & & \multirow{3}{*}{$\frac{\text { Até } 29 \text { tratamentos }}{\mathrm{BC}}$} & \multirow{2}{*}{\multicolumn{2}{|c|}{ De 30 a 100 tratamentos }} & \multirow[t]{3}{*}{ Mínimo } & \multirow[t]{3}{*}{ Máximo } & \multirow[t]{3}{*}{ Amplitude } \\
\hline & & & & & & & \\
\hline & & & $\mathrm{BC}$ & BI & & & \\
\hline$\overline{\mathrm{ACA}}$ & $0,081^{\mathrm{ns}}$ & 40 & 6 & 11 & 15,13 & 108,12 & 92,99 \\
\hline ALT & $0,148 *$ & 69 & 8 & 14 & 1,28 & 17,27 & 15,99 \\
\hline $\mathrm{BF}$ & $0,116^{\text {ns }}$ & 25 & 6 & 6 & 20,91 & 85,73 & 64,82 \\
\hline $\mathrm{BP}$ & 0,119 * & 39 & 6 & 12 & 9,36 & 75,74 & 66,38 \\
\hline ESC & $0,129 *$ & 37 & 4 & 10 & 4,41 & 70,03 & 65,62 \\
\hline FLO & $0,202 *$ & 50 & 5 & 12 & 0,38 & 7,62 & 7,24 \\
\hline MG & $0,128 *$ & 46 & 7 & 13 & 8,43 & 75,97 & 67,54 \\
\hline MP & $0,119 *$ & 40 & 2 & 12 & 9,72 & 74,23 & 64,51 \\
\hline M100 & $0,147^{\text {ns }}$ & 22 & - & - & 3,37 & 42,01 & 38,64 \\
\hline NEV & $0,083^{\text {ns }}$ & 17 & - & - & 17,02 & 42,88 & 25,86 \\
\hline NGC & $0,214 *$ & 22 & - & - & 10,46 & 30,08 & 19,62 \\
\hline NPAN & $0,184 *$ & 31 & - & - & 10,46 & 24,83 & 14,37 \\
\hline PROD & $0,130^{*}$ & 88 & 8 & 14 & 3,78 & 51,36 & 47,58 \\
\hline
\end{tabular}

Tabela 1. Teste de Lilliefors e estatísticas descritivas dos coeficientes de variação dos caracteres estudados referentes a 110 experimentos de arroz de terras altas ${ }^{(1)}$.

(1)ACA: acamamento; ALT: altura da planta; BF: brusone na folha; BP: brusone na panícula; ESC: escaldadura da folha; FLO: floração; MG: mancha dos grãos; MP: mancha parda na folha; M100: massa de 100 grãos; NEV: número de espiguetas vazias; NGC: número de grãos cheios; NPAN: número de

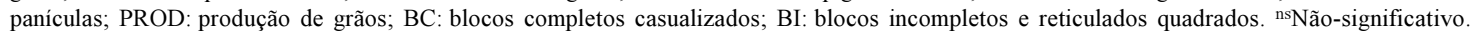
* Significativo a $5 \%$ de probabilidade pelo teste de Lilliefors. 
Para cada caráter, de forma geral e considerando caráter dentro de delineamento, foram obtidos os índices numéricos: mínimo, máximo, amplitude, mediana, amplitude interquartílica, e pseudo-sigma. Foram construídas faixas de classificação de coeficientes de variação de acordo com (1), para todas as variáveis selecionadas, de forma geral, e considerando o delineamento experimental utilizado.

\section{Resultados e Discussão}

Houve grande variabilidade dos dados entre e dentro dos caracteres, e as maiores amplitudes referem-se aos caracteres relacionados às doenças e ao acamamento, o que se explica pelo fato de estarem sujeitos a grande variabilidade, em virtude da influência de vários fatores (Tabela 1). Entre estes, a suscetibilidade do material utilizado; a forma de determinação destes caracteres, que sofre a influência da acuidade visual dos avaliadores, tendo em vista $o$ critério de notas ( 0 a 9 ) adotado para a avaliação, e o número de repetições utilizado, que influi nos valores do CV (Pimentel-Gomes, 1991). Quanto aos caracteres M100, NEV, NGC, NPAN e PROD, os valores de CV máximo foram encontrados nos ensaios com deficiência hídrica do solo. As diferenças observadas justificam a necessidade de uma classificação distinta dos coeficientes de variação de cada caráter, como sugerido em Garcia (1989), Scapim et al. (1995) e Amaral et al. (1997).

Não houve diferenças entre os métodos comparados, quanto à definição dos intervalos dos CVs (Tabela 2). Isto sugere que o método proposto é adequado e eficiente para a definição de faixas de $\mathrm{CV}$, e confirma que quando a distribuição é normal os in- tervalos são muito próximos, uma vez que PS produz uma estimativa do desvio-padrão próximo do desvio-padrão da amostra. É de se esperar que este método também seja tão eficiente quanto o método de Amaral et al. (1997), que é o método de Garcia (1989) com pequena modificação no cálculo da variância dos CVs, e que também considera como exigência básica a distribuição normal dos CVs. Tendo como base estas comparações, pode-se inferir que o método proposto seja mais adequado e muito mais vantajoso de ser usado, uma vez que não há a necessidade de se preocupar com a distribuição dos dados, e pode ser utilizado para qualquer variável que está sendo estudada.

Houve grande variação nos intervalos dos CVs, onde valores considerados como médios e altos seriam considerados respectivamente como altos e muito altos segundo a classificação de Pimentel-Gomes (1985), como é o caso das variáveis relacionadas às doenças e ao acamamento; isto confirma a necessidade de se considerar na classificação dos CVs a natureza da variável estudada (Tabela 3 ).

Foram construídas faixas de classificação dos CVs dos caracteres estudados, considerando não apenas a natureza da variável estudada, mas também o delineamento experimental utilizado nos experimentos com 30 a 100 tratamentos (Tabela 4). Quanto às variáveis M100, NEV, NGC e NPAN, não foi possível fazer uma classificação dos CVs, devido à disponibilidade de dados apenas nos experimentos que utilizaram BC com até 29 tratamentos. Nos intervalos onde foram agrupados mais de um experimento, há experimentos com três ou quatro repetições com tamanho de parcelas variando entre 3,0 e 6,4 $\mathrm{m}^{2}$, cuja

Tabela 2. Intervalo dos coeficientes de variação segundo o método proposto e o de Garcia (1989) para as variáveis que apresentaram distribuição normal ${ }^{(1)}$.

\begin{tabular}{|c|c|c|c|c|c|c|c|}
\hline \multirow[t]{2}{*}{ Variável } & \multirow[t]{2}{*}{ Método } & \multicolumn{6}{|c|}{ Intervalo (\%) } \\
\hline & & \multirow{2}{*}{$\begin{array}{c}\begin{array}{c}\text { Baixo } \\
(\mathrm{CV} \leq)\end{array} \\
29,61(10)\end{array}$} & \multicolumn{2}{|c|}{$\begin{array}{l} \\
\quad(<\mathrm{CV} \leq) \\
\end{array}$} & \multicolumn{2}{|c|}{ 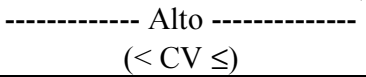 } & \multirow{2}{*}{$\begin{array}{l}\text { Muito alto } \\
(\mathrm{CV}>)\end{array}$} \\
\hline \multirow[t]{2}{*}{$\mathrm{ACA}$} & Proposto & & 29,61 & $74,81(37)$ & 74,81 & $97,41(8)$ & \\
\hline & Garcia (1989) & $29,77(10)$ & 29,77 & $74,21(37)$ & 74,21 & $96,43(8)$ & $96,43(2)$ \\
\hline \multirow[t]{2}{*}{$\mathrm{BF}$} & Proposto & $26,46(9)$ & 26,46 & $58,80(24)$ & 58,80 & $74,97(3)$ & $74,97(1)$ \\
\hline & Garcia (1989) & $25,90(7)$ & 25,90 & $55,84(25)$ & 55,84 & $70,81(3)$ & $70,81(2)$ \\
\hline \multirow[t]{2}{*}{ M100 } & Proposto & $8,70(5)$ & 8,70 & $21,18(13)$ & 21,18 & $27,42(2)$ & $27,42(2)$ \\
\hline & Garcia (1989) & $6,26(5)$ & 6,26 & $24,78(14)$ & 24,78 & $34,04(2)$ & $34,04(1)$ \\
\hline \multirow[t]{2}{*}{ NEV } & Proposto & $23,16(2)$ & 23,16 & $37,26(12)$ & 37,26 & $44,31(3)$ & $44,31(0)$ \\
\hline & Garcia (1989) & $23,66(3)$ & 23,66 & $37,46(11)$ & 37,46 & $44,36(3)$ & $44,36(0)$ \\
\hline
\end{tabular}

(1)ACA: acamamento; BF: brusone na folha; M100: massa de 100 grãos; NEV: número de espiguetas vazias; os valores entre parênteses referem-se ao número de experimentos classificados nos respectivos intervalos. 
variação, considerada pequena, não foi levada em consideração.

Os intervalos dos CVs do grupo BI, em geral, foram menores do que os do grupo $\mathrm{BC}$, o que demonstra também que, quando o número de tratamentos é elevado, o tipo de delineamento experimental é fator importante para a avaliação do CV, o que está em desacordo com as conclusões de Estefanel et al. (1987), e, por conseqüência, também com as de Amaral et al. (1997). Pelo fato de Estefanel et al. (1987) não

Tabela 3. Intervalo dos coeficientes de variação dos caracteres estudados segundo a classificação proposta(1).

\begin{tabular}{|c|c|c|c|c|c|c|}
\hline \multirow[t]{2}{*}{ Variável } & \multicolumn{6}{|c|}{ Intervalo (\%) } \\
\hline & $\begin{array}{l}\text { Baixo } \\
(\mathrm{CV} \leq)\end{array}$ & ---------- & $\begin{array}{l}\text { dio }---------- \\
J \leq)\end{array}$ & ----- & $\begin{array}{l}0 \text { - } \\
V \leq\end{array}$ & $\begin{array}{l}\text { Muito alto } \\
(\mathrm{CV}>)\end{array}$ \\
\hline ACA & $29,61(10)$ & 29,61 & $74,81(37)$ & 74,81 & $97,41(8)$ & $97,41(2)$ \\
\hline ALT & $3,87(11)$ & 3,87 & $7,35(58)$ & 7,35 & $9,09(11)$ & $9,09(11)$ \\
\hline $\mathrm{BF}$ & $26,46(9)$ & 26,46 & $58,80(24)$ & 58,80 & $74,97(3)$ & $74,97(1)$ \\
\hline BP & $19,82(6)$ & 19,82 & $57,16(42)$ & 57,16 & $75,83(9)$ & $75,83(0)$ \\
\hline ESC & $12,97(4)$ & 12,97 & $41,11(36)$ & 41,11 & $55,18(8)$ & $55,18(3)$ \\
\hline FLO & $0,79(3)$ & 0,79 & $3,19(47)$ & 3,19 & $4,39(9)$ & $4,39(8)$ \\
\hline MG & $15,35(5)$ & 15,35 & $42,73(48)$ & 42,73 & $56,42(6)$ & $56,42(7)$ \\
\hline MP & $17,39(4)$ & 17,39 & $49,57(43)$ & 49,57 & $65,66(6)$ & $65,66(1)$ \\
\hline M100 & $8,70(5)$ & 8,70 & $21,18(13)$ & 21,18 & $27,42(2)$ & $27,42(2)$ \\
\hline NEV & $23,16(2)$ & 23,16 & $37,26(12)$ & 37,26 & $44,31(3)$ & $44,31(0)$ \\
\hline NGC & $13,09(5)$ & 13,09 & $18,31(13)$ & 18,31 & $20,92(1)$ & $20,92(3)$ \\
\hline NPAN & $11,23(5)$ & 11,23 & $18,55(19)$ & 18,55 & $22,21(3)$ & $22,21(4)$ \\
\hline PROD & $12,20(12)$ & 12,20 & $27,90(78)$ & 27,90 & $35,75(10)$ & $35,75(10)$ \\
\hline
\end{tabular}

(1)ACA: acamamento; ALT: altura da planta; BF: brusone na folha; BP: brusone na panícula; ESC: escaldadura da folha; FLO: floração; MG: mancha dos grãos; MP: mancha parda na folha; M100: massa de 100 grãos; NEV: número de espiguetas vazias; NGC: número de grãos cheios; NPAN: número de panículas; PROD: produção de grãos; os valores entre parênteses referem-se ao número de experimentos classificados nos respectivos intervalos.

Tabela 4. Intervalo dos coeficientes de variação segundo a classificação proposta, agrupados por delineamento experimental utilizando 30 a 100 tratamentos $^{(1)}$.

\begin{tabular}{|c|c|c|c|c|c|c|c|}
\hline \multirow{3}{*}{$\begin{array}{l}\text { Variável } \\
\text { ACA }\end{array}$} & \multirow{3}{*}{$\begin{array}{r}\text { Delineamento } \\
\text { experimental } \\
\text { BC }\end{array}$} & \multicolumn{6}{|c|}{ Intervalo (\%) } \\
\hline & & \multirow{2}{*}{$\begin{array}{l}\text { Baixo } \\
(\mathrm{CV} \leq) \\
36,86(2)\end{array}$} & \multicolumn{2}{|c|}{$\begin{array}{l}----- \\
\quad(<\mathrm{CV} \leq)\end{array}$} & \multicolumn{2}{|c|}{$\begin{array}{l}-(<\mathrm{CV} \leq) \\
\end{array}$} & \multirow{2}{*}{$\begin{array}{l}\text { Muito alto } \\
(\mathrm{CV}>)\end{array}$} \\
\hline & & & 36,86 & $67,42(4)$ & 67,42 & $82,70(0)$ & \\
\hline & BI & $13,93(0)$ & 13,93 & $62,77(7)$ & 62,77 & $87,19(4)$ & $87,19(0)$ \\
\hline \multirow[t]{2}{*}{ ALT } & $\mathrm{BC}$ & $5,21(1)$ & 5,21 & $7,69(6)$ & 7,69 & $8,93(1)$ & $8,93(0)$ \\
\hline & BI & $4,21(3)$ & 4,21 & $6,01(9)$ & 6,01 & $6,91(1)$ & $6,91(1)$ \\
\hline \multirow[t]{2}{*}{$\mathrm{BF}$} & $\mathrm{BC}$ & $22,01(0)$ & 22,01 & $33,27(4)$ & 33,27 & $38,90(1)$ & $38,90(1)$ \\
\hline & BI & $40,45(0)$ & 40,45 & $45,79(4)$ & 45,79 & $48,46(1)$ & $48,46(1)$ \\
\hline \multirow[t]{2}{*}{ BP } & $\mathrm{BC}$ & $21,10(1)$ & 21,10 & $51,24(4)$ & 51,24 & $66,31(1)$ & $66,31(0)$ \\
\hline & BI & $20,91(1)$ & 20,91 & $63,07(11)$ & 63,07 & $84,15(0)$ & $84,15(0)$ \\
\hline \multirow[t]{2}{*}{$\mathrm{ESC}$} & $\mathrm{BC}$ & $4,72(0)$ & 4,72 & $65,52(4)$ & 65,52 & $95,92(0)$ & $95,92(0)$ \\
\hline & BI & $10,64(0)$ & 10,64 & $30,76(7)$ & 30,76 & $40,82(2)$ & $40,82(1)$ \\
\hline \multirow[t]{2}{*}{ FLO } & $\mathrm{BC}$ & $1,25(1)$ & 1,25 & $2,61(3)$ & 2,61 & $3,29(0)$ & $3,29(1)$ \\
\hline & BI & $1,30(2)$ & 1,30 & $2,60(7)$ & 2,60 & $3,25(2)$ & $3,25(1)$ \\
\hline \multirow[t]{2}{*}{ MG } & $\mathrm{BC}$ & $22,64(1)$ & 22,64 & $45,14(5)$ & 45,14 & $56,39(1)$ & $56,39(0)$ \\
\hline & BI & $21,78(2)$ & 21,78 & $39,92(8)$ & 39,92 & $48,99(2)$ & $48,99(1)$ \\
\hline \multirow[t]{2}{*}{ MP } & $\mathrm{BC}$ & $18,57(1)$ & 18,57 & $23,49(0)$ & 23,49 & $25,95(1)$ & $25,95(0)$ \\
\hline & BI & $14,52(0)$ & 14,52 & $35,74(9)$ & 35,74 & $46,35(0)$ & $46,35(3)$ \\
\hline \multirow[t]{2}{*}{ PROD } & $\mathrm{BC}$ & $13,18(1)$ & 13,18 & $43,70(6)$ & 43,70 & $58,96(1)$ & $58,96(0)$ \\
\hline & BI & $10,87(0)$ & 10,87 & $20,79(11)$ & 20,79 & $25,75(3)$ & $25,75(0)$ \\
\hline
\end{tabular}

(1)ACA: acamamento; ALT: altura da planta; BF: brusone na folha; BP: brusone na panícula; ESC: escaldadura da folha; FLO: floração; MG: mancha dos grãos; MP: mancha parda na folha; PROD: produção de grãos; BC: blocos completos casualizados; BI: blocos incompletos e reticulados quadrados; os valores entre parênteses referem-se ao número de experimentos classificados nos respectivos intervalos. 
terem estudado o efeito do delineamento considerando simultaneamente o número de tratamentos empregado, este efeito pode não ter sido evidenciado dentro do material por eles estudado.

$\mathrm{O}$ uso de $\mathrm{BC}$ é apropriado quando o número de tratamentos é pequeno; entretanto, se esse número é grande, como nesta situação, a adoção de $\mathrm{BC}$ pode resultar em uma elevação do erro experimental, em virtude do aumento do tamanho dos blocos, tendo como conseqüência CVs mais altos. Aspectos práticos sobre a formação de blocos no campo são discutidos em Mead (1988). Nas situações onde o número de tratamentos é elevado, a utilização do BI reduz o erro experimental, uma vez que este delineamento consegue acomodar um grande número de tratamentos em pequenos blocos, mais homogêneos, mesmo em detrimento de se usar um menor número de repetições, embora o número de repetições possa influir também na correta avaliação da precisão dos experimentos (Pimentel-Gomes, 1991; Amaral et al., 1997). A maior dificuldade de análise dos delineamentos em blocos incompletos, como também a maior perda de graus de liberdade no resíduo, são freqüentemente compensados por uma grande redução no quadrado médio residual, de sorte que, não raro, obtêm-se experimentos mais precisos e mais eficientes (Patterson \& Hunter, 1983; Pimentel-Gomes, 1985; Kempton et al., 1994).

As tabelas aqui apresentadas devem ser utilizadas como referência pelo pesquisador, para verificar se os resultados de CVs obtidos, em experimentação com arroz de terras altas, estão, ou não, dentro de uma faixa esperada de valores.

\section{Conclusões}

1. O método proposto é eficiente para a definição de faixas dos coeficientes de variação, independentemente da distribuição do coeficientes de variação.

2. Na avaliação dos coeficientes de variação, em experimentos com elevado número de tratamentos, é importante considerar o delineamento experimental utilizado.

3. Variáveis relacionadas às doenças e ao acamamento, e experimentos com número grande de tratamentos em BC, apresentam altos coeficientes de variação.

\section{Referências}

AMARAL, A. M. do; MUNIZ, J. A.; SOUZA, M. de. Avaliação do coeficiente de variação como medida da precisão na experimentação com citros. Pesquisa Agropecuária Brasileira, Brasília, v. 32, n. 12, p. 12211225, dez. 1997.

BLANXART, M. F.; COSIALLS, L. S.; OLMOS, J. G.; PUIG, R. F.; OSET, J. T. Análisis exploratorio de datos: nuevas técnicas estadísticas. Barcelona: Promociones y Publicaciones Universitarias, 1992. 296 p.

CAMPOS, H. de. Estatística aplicada à experimentação com cana-de-açúcar. Piracicaba: Fealq, 1984. 292 p.

CAMPOS, H. de. Estatística experimental nãoparamétrica. 3. ed. Piracicaba: USP, 1979. 343 p.

CHAVES, L. J. Tamanho de parcela para seleção de progênies de milho (Zea mays L.). Piracicaba: Esalq, 1985. 148 p. Tese de Doutorado.

CONOVER, W. J. Practical nonparametric statistics. New York: J. Wiley, 1971. 302 p.

COSTA, N. H. A. D. Um método de classificação de coeficientes de variação para a cultura do arroz de terras altas. Goiânia: UFG, 2000. 32 p. Monografia de especialização em estatística.

CUNHA, D. F. da. Efeitos das transformações de dados sobre o coeficiente de variação. Revista de Agricultura, Piracicaba, v. 75, n. 3, p. 323-338, dez. 2000.

EMBRAPA. Centro Nacional de Pesquisa de Arroz e Feijão (Goiânia, GO). Embrapa e parceiros lançam 85 cultivares de arroz em 15 anos de pesquisa. Pesquisa em Foco, Goiânia, n. 4, p. 1-2, out. 1997.

ESTEFANEL, V.; PIGNATARO, I. A. B.; STORCK, L. Avaliação do coeficiente de variação de experimentos com algumas culturas agrícolas. In: SIMPÓSIO DE ESTATÍSTICA APLICADA À EXPERIMENTAÇÃO AGRONÔMICA, 2., 1987, Londrina. Anais... Londrina: UEL/Região Brasileira da Sociedade Internacional de Biometria, 1987. p. 115-131.

GARCIA, C. H. Tabelas para classificação do coeficiente de variação. Piracicaba: Ipef, 1989. 12 p. (Circular Técnica, 171).

HOAGLiN, D. C.; MOSTELLER, F.; TUKEY, J. W. Understanding robust and exploratory data analysis. New York: J. Wiley, 1983. 447 p. 
JUDICE, M. G.; MUNIZ, J. A.; CARVALHEIRO, R. Avaliação do coeficiente de variação na experimentação com suínos. Ciência e Agrotecnologia, Lavras, v. 23, n. 1, p. 170-173, jan./mar. 1999.

KEMPTON, R. A.; SERAPHIN, J. C.; SWORD, A. M. Statistical analysis of two-dimensional variation in variety yield trials. Journal of Agricultural Science, Cambridge, Inglaterra, v. 3, n. 122, p. 335-342, 1994.

MEAD, R. The design of experiments. Cambridge, Inglaterra: Cambridge University Press, 1988. cap. 2.

PATTERSON, H. D.; HUNTER, E. A. The efficiency of incomplete block designs in national list and recommended list cereal variety trials. Journal of Agricultural Science, Cambridge, Inglaterra, v. 4, n. 101, p. 427-433, 1983.

PIMENTEL-GOMES, F. Curso de estatística experimental. 11. ed. Piracicaba: Esalq, 1985. 467 p.
PIMENTEL-GOMES, F. O índice de variação: um substituto vantajoso do coeficiente de variação. Piracicaba: Ipef, 1991. 4 p. (Circular Técnica, 178).

SCAPIM, C. A.; CARVALHO, C. G. P. de; CRUZ, C. D. Uma proposta de classificação dos coeficientes de variação para a cultura do milho. Pesquisa Agropecuária Brasileira, Brasília, v. 30, n. 5, p. 683-686, maio 1995.

SPIEGEL, M. R. Estatística. 3. ed. São Paulo: Makron Books, 1993. 643 p. (Coleção Schaum).

STEEL, R. G. D.; TORRIE, J. H. Principles and procedures of statistics: a biometrical approach. 2. ed. New York: McGraw-Hill, 1980. 633 p.

TUKEY, J. W. Exploratory data analysis. Reading: Addison-Wesley, 1977. 688 p. 